\title{
Spectrum of Clinical Manifestations of Canine Distemper in Dogs
}

\author{
M. Geetha* and G. Selvaraju \\ Department of Veterinary Public Health and Epidemiology, Veterinary College and Research \\ Institute, Namakkal - 637 002, Tamil Nadu Veterinary and Animal Sciences University, \\ Chennai - 600 051, India \\ *Corresponding author
}

\section{A B S T R A C T}

\section{Keywords}

Canine Distemper,

Dogs, Clinical manifestations

\section{Article Info}

Accepted:

15 November 2019

Available Online:

10 December 2019
Canine distemper is a most common highly contagious viral disease of domestic dogs, wild carnivores causing higher morbidity and mortality. Forty nine dogs of various age groups were brought to the Infectious disease unit of Veterinary College and Research Institute, Namakkal with the history of clinical manifestations including pyrexia, congested conjunctival mucous membranes, abdominal pustules, purulent ocular and nasal discharge, continuous or intermittent seizures, temporal twitching and a few of them had haemorrhagic gastroenteritis. All dogs were diagnosed by using DFAT using anti-CDV antibody - Fluorescein isothiocyanate (FITC) conjugate. Purulent ocular, pyrexia, abdominal pustules were noticed initially followed by purulent nasal discharge and haemorrhagic gastroenteritis. Majority of the dogs showed chorea, flexor spasms, generalised myoclonus and chewing gum seizures. One puppy had intermittent seizures and one dog had enamel hypoplasia with irregular dentine surface. Regular immunization of bitches, proper feeding of colostrum to puppies, periodical immunization of puppies from four weeks of age depending upon their colostrum recipient status will definitely protect the dogs against Canine Distemper.

\section{Introduction}

Canine distemper (CD) is a highly contagious viral infection of dogs' endemic in India and throughout the world causing huge mortality rates in dogs next to rabies (Deem et al., 2000). The disease is caused by Canine distemper virus (CDV), a member of genus morbilli virus, Paramyxoviridae (Carvalho et al., 2012) affecting wide range of domestic and wild carnivores including dogs, wild dogs, foxes, jackals, hyenas (Van de Bildt et al., 2002), lions, tigers, leopards etc. (Appel et al., 1994). The virus is a small, enveloped, nonsegmented single-stranded, negative-sense RNA virus (about 15,000 bases long) that encodes 6 structural proteins: the nucleocapsid (N) protein, 2 transcriptase-associated proteins (phosphoprotein $\mathrm{P}$ and large protein $\mathrm{L}$ ), the envelope stabilizing matrix (M) protein, and 2 
transmembrane glycoproteins embedded in the viral envelope, which are important immunogens of CDV, the hemagglutinin $(\mathrm{H})$ and fusion (F) proteins (Martella et al., 2008). The CDV is a pantrophic virus targeting respiratory, gastrointestinal, cutaneous, nervous systems and eye of domestic dogs which are immunologically naive. After viral entry, it targets the lymphatic system causes primary viraemia. Followed by primary viraemic phase, the affected dogs exhibit non specific clinical signs including pyrexia, purulent ocular discharge, abdominal pustules and anorexia. Nervous complications are usually noticed in adult dogs without typical prior clinical manifestations. This paper deals with typical clinical manifestations of CD (Appel et al., 1982). This paper describes the spectrum of clinical manifestations of $\mathrm{CD}$ in dogs in Namakkal region of Tamil Nadu.

\section{Materials and Methods}

A total of forty nine dogs were brought to the infectious disease unit of Veterinary Clinical Complex, Veterinary College and Research Institute, Namakkal, Tamil Nadu, India with variable clinical manifestations including pyrexia, congested conjunctival mucous membranes, abdominal pustules, purulent ocular and nasal discharge, continuous or intermittent seizures, temporal twitching and a few of them had haemorrhagic gastroenteritis. Forty five dogs showed various nervous manifestations of $\mathrm{CD}$ including generalised myoclonus, hypermetria, chorea (temporal twitching) and flexar spasms without any previous illness history. Two dogs showed enamel hypoplasia, hyper keratosis of food pads and muzzle. Ocular discharges of the animals were collected aseptically in a sterile swab and smears were made for demonstration of CDV using direct flourescent antibody test (DFAT) for confirmatory diagnosis as per the protocol recommended by Kapil and Neel (2015). Forty seven among forty nine dogs presented were not immunized and most of the owner's were unaware about the immunization of dogs. One puppy had shown intermittent seizures with abdominal pustules.

\section{Results and Discussion}

Canine distemper is one of the serious disease of unimmunized and improperly immunized dogs and its clinical manifestations depending upon the immune status of the dogs exposed to CDV infection and the virus has affinity for epithelial, lymphatic, nervous and endocrine systems (Kapil and Yeary, 2011). Canine distemper viral infection in dogs presents with multiple clinical courses and outcome of CDV infection ranges from complete recovery to persistent infection leading to death depending on the age and immune status of the infected animals (Martella et al., 2008). Clinical signs of CD are often unapparent or initially mild during initial phase of fever is characterized by mucopurulent oculonasal discharges, conjunctivitis, respiratory distress, anorexia, vomiting, diarrhea and dehydration, and cutaneous rash (Appel et al., 1982). The findings of the above author is well in accordance with the clinical signs of $\mathrm{CD}$ affected dogs in this study including purulent ocular discharge (Plate. 1), abdominal pustules (Plate. 2) and purulent nasal discharge (Plate. 3). All the dogs presented with the above clinical signs were not either unimmunized or improper immunization history (only with primary dose of multivalent vaccines containing CDV, Canine adenovirus 1 and 2, Canine parvovirus and Leptospira Icterohaemorrhagiae and L. Canicola). Lack of complete or partial immunity against CDV may be responsible for the variant clinical signs of $C D$ in dogs due pathophysiology mechanisms associated with the disease occurrence. All dogs were treated with broad spectrum antibiotic (Amoxycillin and cloxacillin @ $20 \mathrm{mg} / \mathrm{kg}$ body weight to counteract against secondary bacterial 
complications and supportive therapy with Vitamin B1, B6 and B12).

Generalised myoclonus, hypermetria, chorea, chewing gum seizures and flexor spasms were noticed either alone or in combination in forty five dogs and hyper keratinization of foot pads and muzzle was noticed in two dogs presented for treatment (Plate. 4 \& 5). This is in correlation with the findings of Beineke et al., (2009) who reported that weak humoral and cell-mediated responses lead to systemic

Plate.1 Purulent ocular discharge in a dog with CDV infection

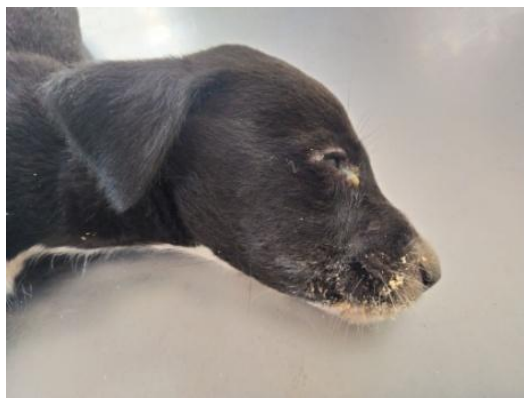

Plate.3 Purulent nasal discharge - Canine distemper in $\operatorname{dog}$



Plate.5 Hyperkeratinzation of muzzle in dog affected with CDV

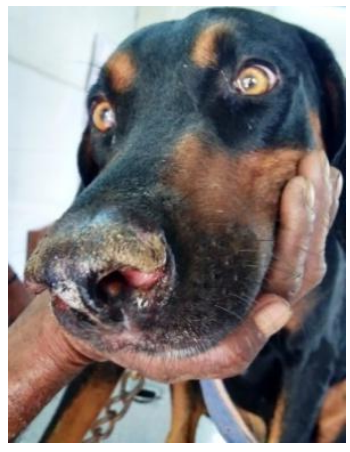

intracellular spread of virus to the epithelial cells of the gastrointestinal and urinary tracts, skin, and the endocrine and central nervous systems causing direct virus-mediated damage. Additional clinical signs that may occur are localized twitching, ascending paresis/paralysis, and/or convulsions. The infection may either prove fatal or persist resulting in subacute or chronic central nervous system (CNS) signs (Beineke et al., 2009).

Plate.2 Canine distemper affected dogs with abdominal pustules

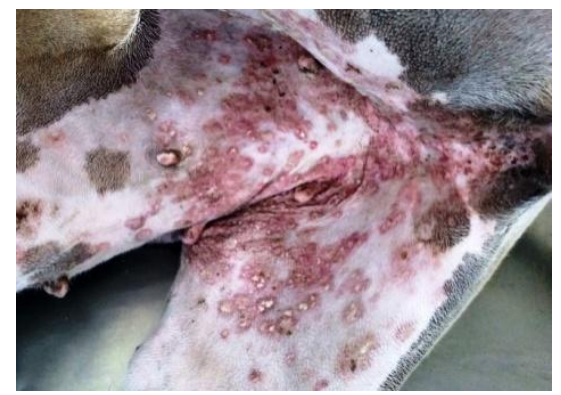

Plate.4 Hyper keratinization of foot pad

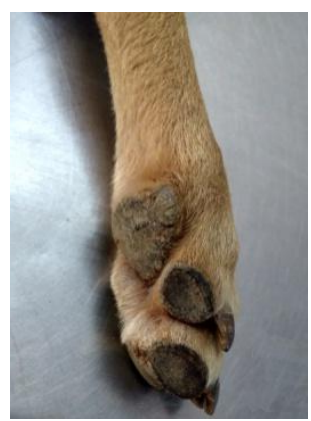

Plate.6 Enamel hypoplasia and irregular dentine in a dog affected with CD

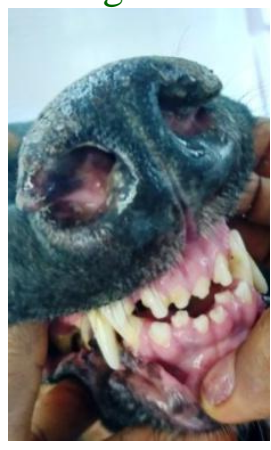


An unimmunized Dobermann dog of four year age showed chewing gum seizures, chorea and showed irregular appearance of enamel and dentine (Plate. 6). Greene and Vandevelde (2012) described that young puppies infected with CDV before the eruption of permanent dentition can cause severe damage to enamel, dentine or roots of their teeth. Enamel or dentine can show irregular appearance. Enamel hypoplasia with or without neurological signs may be an incidental finding in an older $\operatorname{dog}$ and is relatively pathognomonic for prior infection with CDV. The report of the above author is in correlation with the findings of this study wherein irregular appearance of dentine was observed along with nervous signs indicate that the dog was infected with CDV in its early part of its life.

Canine distemper is a most common highly contagious viral disease of dogs of all age groups. Clinical disease occurrence is always related to the immune status of dogs. Priming of colostrum recipient puppies with multivalent vaccines containing $\mathrm{CD}$ antigen at sixth week age, later boosters once in two to three weeks up to 16 weeks of age, followed by booster after a year and boosters once in three years will give a protective immunity to puppies against this deadliest viral infection.

For colostrum deprived puppies immunization should be started as early as four weeks of age but at most care should be taken to give live attenuated vaccines for puppies of less than four weeks age for providing a wide immune spectrum against CD infection. Proper colostrum feeding of puppies, regular immunization of bitches before breeding with live attenuated $C D$ vaccines will definitely helpful in prevention of CD in dogs.

\section{References}

Appel, M. J. G., W. R. Shek, and B. A. Summers, 1982. Lymphocyte- mediated immune cytotoxicity in dogs infected with virulent canine distemper virus. Infection and Immunity, (37): 2, 592-600.

Appel, M. J., R. A. Yates, G. L. Foley, J.J. Bernstein, S. Santinelli, L.H. Spelman, L.D. Miller, L.H. Arp, M.Anderson, M.Barr et al., 1994. Canine distemper epizootic in lions, tigers, and leopards in North America. J. Vet. Diagn. Invest., 6, 277-288.

Beineke, A., C. Puff, F. Seehusen, and W. Baumgartner. 2009. Pathogenesis and immunopathology of systemic and nervous canine distemper. Vet Immunol Immunopathol., 127(1-2):118.

Carvalho, O.V., C. V. Botelho, C. G. Torres Ferreira, P. O. Scherer, J.A.P.S. Martins, M. R. Almeida and A. S. Junior. 2012. Immunopathogenic and neurological mechanisms of Canine Distemper virus. Advances in Virology. doi:10.1155/2012/163860.

Deem, S.L., L. H. Spelman, R. A. Yates and R. J. Montali, 2000. Canine Distemper in terrestrial carnivores: A review. Journal of Zoo and Wildlife Medicine 31:(4), 441-451.

Greene,G. and N. Decaro, 2012. Canine distemper. In: Infectious diseases of the dog and cat. Fourth edition, Greene, G., (Eds.). Elsevier Saunders, Missouri, USA. pp. 25-42.

Kapil, S. and T. J. Yeary, 2011. Canine Distemper spillover in domestic dogs from urban wildlife. Vet Clin Small Anim., 41: 1069-1086.

Kapil, S. and T. Neel. 2015. Canine distemper virus antigen detection in external epithelia of recently vaccinated, sick dogs by fluorescence microscopy is a valuable prognostic indicator. J Clin Microbiol., 53:687-691. doi:10.1128/JCM.02741-14.

Martella, V., G. Elia and C. Buonavoglia, 
2008. Canine distemper virus. Vet Clin North Am Small Anim Pract., 38: 78797.

Van de Bildt, M. W. G., T. Kuiken, A. M. Visee, S. Lema, T. R. Fitzjohn, and A.
D.M. E. Osterhaus, 2002. Distemper outbreak and its effect on African wild dog conservation. Emerging Infectious Diseases. (8): 2, 211-213.

\section{How to cite this article:}

Geetha, M. and Selvaraju, G. 2019. Spectrum of Clinical Manifestations of Canine Distemper in Dogs. Int.J.Curr.Microbiol.App.Sci. 8(12): 1916-1920. doi: https://doi.org/10.20546/ijcmas.2019.812.229 